\title{
Effect of Phytase Enzyme and Phosphatic Fertilizers on Available Phosphorus in an Alfisol
}

\author{
S. T. Shinde, A. B. Jadhav", A. V. Patil, A. L. Pharande, G. D. Patil, \\ B. D. Tamboli and J. M. Khire \\ Department of Soil Science and Agricultural Chemistry, College of Agriculture, \\ Pune-411 005, Maharashtra, India
}

\begin{abstract}
An experiment involving sixteen treatments with four levels of phytase ( 0 , 240, 480, $720 \mathrm{IU})$ and four levels of phosphorus $\left(0,25,50,100 \mathrm{~kg} \mathrm{~h}^{-1}\right)$ was carried out during kharif 2017 to assess the availability of phosphorus at $0,15,30,45,60$ and 90 days (DAI) after incubation. There was an increase in soil available phosphorus up to $45^{\text {th }}$ days of incubation either with the application of phytase or $\mathrm{P}$ and it reduced after 60 days of incubation. Significantly higher (12.98 $\left.\mathrm{kg} \mathrm{ha}^{-1}\right)$ available phosphorus was observed with the application of phytase @ 720 IU (16.14 kg ha $\left.{ }^{-1}\right)$ and phosphatic fertilizer@100 kg ha ${ }^{-1}$ within 24 hours of incubation over initial available $\mathrm{P}\left(5.20 \mathrm{~kg} \mathrm{ha}^{-1}\right)$. Application of $480 \mathrm{IU}$ phytase resulted in significantly higher available P (51.98 $\mathrm{kg} \mathrm{ha}^{-1}$ ) which was at par with $720 \mathrm{IU}$ (50.45 kg ha $\left.{ }^{-1}\right)$. However, combined application of either 480 or $720 \mathrm{IU}$ phytase along with either 50 or $100 \mathrm{~kg} P$ $\mathrm{ha}^{-1}$ through single super phosphate (SSP) had in statistically at par results for available phosphorus. Higher cumulative available phosphorus was recorded with the application of phytase @ $720 \mathrm{IU}$ along with $100 \mathrm{~kg} \mathrm{P} \mathrm{ha}^{-1}$ through SSP $(247.23 \mathrm{~kg}$ $\left.\mathrm{ha}^{-1}\right)$ throughout the incubation period. Further phosphorus release potential indicated that application of phytase @ 720 IU along with $100 \mathrm{~kg} \mathrm{Pha}^{-1}$ through SSP mobilized $0.65 \mathrm{~kg} \mathrm{ha}^{-1}$ day $^{-1}$ phosphorus.
\end{abstract}

Key words: Phytase, phosphatic fertilizer, available phosphorus, $P$ release potential

\section{Introduction}

The availability of $\mathrm{P}$ in soil is influenced by $\mathrm{pH}$ of the soil. In case of soils with low $\mathrm{pH}$, applied soluble phosphorus get converted into insoluble complexes of Fe and Al phosphates (Yadav and Verma 2012). However, in case of soils with slightly acidic $\mathrm{pH}$, soluble phosphorus gets converted into insoluble complexes of $\mathrm{Fe}$ and $\mathrm{Al}$ hydroxides. The availability of soil phosphorous (potential or applied) is largely governed by micro flora and soil enzymes. Acid alkaline phosphatase and phytase are the soil enzymes mainly

*Corresponding author: (Email: abjadhav1234@rediffmail.com) involved in the mineralization of fixed phosphorous. The acid phosphatase and alkaline phosphatase are predominant in acid and alkaline soil respectively (Yadav and Tarafdar 2003). Phytin is an organic form of phosphorus present in plant, soil and organic matter which is hydrolyzed by phytase enzyme. In soil, phosphorus exists both in organic and inorganic form, the organic phosphorus compounds can be classified into i) the inositol phosphate, primarily of plant origin up to $60 \%$ of soil organic phosphorus, ii) the nucleic acids and iii) phospholipids (Aseri et al. 2009). In soils, 20-85 $\%$ of the total phosphorus remains in organic form, but 
plants can only utilize the organic phosphorus after its mineralization. Phytases are synthesized by many microorganisms, including various bacteria, fungi, micromycetes and other microbes which help to increase soil phosphorus availability for plant nutrition (Aseri et al. 2009). The importance of soil organic phosphorus as a source of plant available phosphorus depends on its rate of solubilisation and release of inorganic phosphorus. Phosphatase and phytase catalyzes hydrolytic cleavage of the C-O-P ester bond of organic phosphorus present in the soil and release plant available inorganic form $\left(\mathrm{HPO}_{4}^{-2}\right.$ and $\mathrm{H}_{2} \mathrm{PO}_{4}{ }^{-}$) (Yadav and Tarafdar 2003).

Till date scanty information is available on the use of different levels of phytase and inorganic phosphorus in an Alfisols of Konkan region and hence, the present investigation was undertaken with objectives to assess the effect of phytase and phosphatic fertilizer levels on periodical phosphorus availability and its release potential.

\section{Materials and Methods}

An incubation (0, 15, 30, 45 and 90 days) study (pot culture) was conducted at Division of Soil Science and Agricultural Chemistry, College of Agriculture, Pune during kharif 2017 to assess the effect of phytase enzyme and phosphatic fertilizers on available phosphorus in a surface soil $(0-15 \mathrm{~cm})$. The experiment having sixteen treatments with four levels of phytase enzyme (@0,240,480 and 720 IU) and four levels of phosphatic fertilizers (@0,25,50 and100 kg ha ${ }^{-1}$ )was replicated thrice in Factorial Completely Randomized Design. The initial physio-chemical and biochemical properties of soil were analyzed using standard methods. The phytase enzyme isolated from Aspergillus niger by National Collection of Industrial Microorganisms (NCIM), National Chemical Laboratory, Pune, India was procured for present study. The enzyme unit was expressed as IU, which is the amount of enzyme that liberated $1 \mu \mathrm{mol}$ of inorganic orthophosphate from phytin per minute at $\mathrm{pH} 5.5$ at $37^{\circ} \mathrm{C}$. The plastic pots (288) with an inner diameter of 12 $\mathrm{cm}$ were filled with $200 \mathrm{~g}$ soil $(2.0 \mathrm{~mm})$. Treatment-wise quantity of phytase enzyme and phosphatic fertilizers (after dilution) were mixed in soil. In order to apply phytase@240,480 and 720 IU, the procured phytase @ $2.6 \mathrm{~g}$ was dissolved in 1 liter water to have 1 lakh IU stock solution. From this stock solution, working standards@115.2, 230.4 and $345.6 \mathrm{ml}$ were pipetted and volume was made to $480 \mathrm{ml}$ and applied @ $10 \mathrm{ml}$ to 48 pots to have 240, 280, 720 IU phytase enzyme respectively, and for control only de-ionised water was applied. In order to provide 25,50 and $100 \mathrm{~kg} \mathrm{ha}^{-1} \mathrm{P}$ through phosphatic fertilizer 669.5, 1339.2 and 2678.4 mg of SSP were weighed, dissolved and volume made to $480 \mathrm{ml}$ and applied @ $10 \mathrm{ml}^{\text {pot }}{ }^{-1}$ respectively to 48 pots. Treatment-wise phytase enzyme and P (through SSP) was thoroughly mixed in soil. At each incubation period $(0,15,30,45,60$ and 90 days) 48 pots were used for assessing the availability of $\mathrm{P}$. The soil available phosphorus was assessed periodically by Bray's and Kurtz (1945) method.

The phosphorus release potential $\left(\mathrm{kg} \mathrm{ha}^{-1}\right.$ day $\left.^{-1}\right)$ was calculated and presented (Table 6) for each incubation period using following mathematical expression by considering respective incubation days.
Phosphorus release potential $\left(\mathrm{kg} \mathrm{ha}^{-1}\right.$ day $\left.^{-1}\right)=$

Where, $X=15,30,45,60$ and 90

\section{Results and Discussion}

Availability of phosphorus was significantly influenced by application of phytase and phosphatic fertilizer at all the incubation periods. The combined application of higher level of phytase and inorganic $\mathrm{P}$ resulted in higher soil available phosphorus. Results revealed that significantly higher available phosphorus was noticed with the application of phytase @ 720 IU (16.14 kg ha ${ }^{-1}$ ) and phosphatic fertilizer @ $100 \mathrm{~kg} \mathrm{ha}^{-1}$ 
within 24 hours of incubation over initial value of 5.20 $\mathrm{kg} \mathrm{ha}^{-1}$ (Table 1). However, combined application of phytase@720 IU + $100 \mathrm{~kg} \mathrm{ha}^{-1} \mathrm{P}$ recorded significantly higher soil available $\mathrm{P}\left(17.10 \mathrm{~kg} \mathrm{ha}^{-1}\right)$ than other interactions at zero day of incubation. There was increase in available phosphorus at $15^{\text {th }}$ and $30^{\text {th }}$ days of incubation with phytase@720 IU while it was higher at $45^{\text {th }}$ day of incubation in the pots receiving 480 IU phytase. Application of $100 \mathrm{~kg} \mathrm{P} \mathrm{ha-1} \mathrm{recorded} \mathrm{higher}$ phosphorus available at $15^{\text {th }}$ and $30^{\text {th }}$ days of incubation than other treatments but at $45^{\text {th }}$ DAI, application of 50 $\mathrm{kg} \mathrm{P} \mathrm{ha}{ }^{-1}$ recorded higher phosphorus availability (Table 2).

The individual effects of phytase and phosphatic fertilizer levels on periodical mean soil available phosphorus is presented in table 3 and 4 . It was noticed that there was consistent increase in soil available phosphorus due to application of increasing levels of phytase or phosphatic fertilizer from 0 to $45^{\text {th }}$ day of incubation but at $60^{\text {th }}$ and $90^{\text {th }} \mathrm{DAI}$, slight decrease in phosphorus availability was noticed for $480 \mathrm{IU}$ phytase as well as $50 \mathrm{~kg} \mathrm{ha}^{-1} \mathrm{P}$ and it was statistically at par with the application of phytase @ 720 IU and $100 \mathrm{~kg}$ $\mathrm{P} \mathrm{ha}^{-1}$. The application of higher levels of phytase @ 480 or 720 IU was more effective in releasing the phosphorus during early incubation periods ( 15 day and 30 day) than the application of higher levels of P@ $90 \mathrm{~kg}$ $\mathrm{ha}^{-1}$ and $100 \mathrm{~kg} \mathrm{ha}^{-1}$. The reduction in soil available phosphorus at $45^{\text {th }}, 60^{\text {th }}$ and $90^{\text {th }}$ DAI was more pronounced with 720 IU phytase. The availability of soil-P was higher at initial incubation periods 0,15 days and 30 DAI with the application of phytase @ 720 IU than 480 IU at 0,15 and 30 DAI.

There was an increase in available phosphorus up to $45^{\text {th }}$ DAI in all the treatments of phytase and P. Application of phytase @ 480 IU with $100 \mathrm{~kg} \mathrm{ha}^{-1} \mathrm{P}$ had highest available phosphorus at $45^{\text {th }}$ DAI followed by phytase@240 IU with 50 kg ha ${ }^{-1} \mathrm{P}$. The cumulative available phosphorus was maximum (247.23 $\left.\mathrm{kg} \mathrm{ha}^{-1}\right)$ with the application of phytase @ 720 IU with $100 \mathrm{~kg} \mathrm{ha}^{-}$ ${ }^{1} \mathrm{P}($ Table 5).

The $\mathrm{P}$ release potential was found to be maximum $\left(0.65 \mathrm{~kg} \mathrm{ha}^{-1} \mathrm{day}^{-1}\right)$ with the application of phytase@720 IU + $100 \mathrm{~kg} \mathrm{ha}^{-1} \mathrm{P}$ followed by application of phytase@480 IU with 50 kg ha ${ }^{-1} \mathrm{P}$. Lowest $\left(0.36 \mathrm{~kg} \mathrm{ha}^{-1}\right)$ available phosphorus potential per day was recorded with application of phytase @ 0 IU + $25 \mathrm{~kg} \mathrm{ha}^{-1} \mathrm{P}$.

In acid soils, precipitation of phosphorus as $\mathrm{Fe}$ phosphate $\left(\mathrm{FePO}_{4} \cdot 2 \mathrm{H}_{2} \mathrm{O}\right)$ and $\mathrm{Al}$ phosphate $\left(\mathrm{AlPO}_{4} \cdot 2 \mathrm{H}_{2} \mathrm{O}\right)$ or by adsorption to $\mathrm{Fe}$ and $\mathrm{Al}$ oxides (labile inorganic P) govern the availability of phosphorus. Phosphate solubilizing microorganisms play an important role in releasing plant available inorganic phosphate into soil from unavailable inorganic P (Ca-P, Fe-P, Al-P) through secretion of organic acids (Subba Rao 1995). Prasad and Ram (1986) found higher available $P$ in the rhizophere soil of greengram inoculated with Rhizobium than the uninoculated soil. Gujar et al. (2013) also reported that microbial released phytase had high efficiency to mineralize $\mathrm{P}$ in soils. The organic acids released by phosphorus solubilizing organisms chelates the cations like $\mathrm{Al}$ and $\mathrm{Fe}$ or lower the $\mathrm{pH}$ and compete with phosphate for adsorption sites in soil (Whitelaw 1999). This may be the reason for higher phosphorus in phytase treated pots. The decrease in available phosphorus could be attributed to increase in fungal biomass resulting in enhanced uptake of soluble phosphate for their growth (Coutinho etal. 2012).

\section{Conclusions}

The application of phytase either @ 480 or 720 IU along with phosphatic fertilizers @ 50 or $100 \mathrm{~kg} \mathrm{ha}^{-1}$ were found to be beneficial for availability of phosphorus in an Alfisol up to $45^{\text {th }}$ DAI. Similar levels of phytase and phosphatic fertilizer were found superior for cumulative available $\mathrm{P}$ with per day release potential. 
Table 1. Physico-chemical properties of initial soil

\begin{tabular}{lc}
\hline Parameter & Values \\
\hline $\mathrm{pH}(1: 2.5)$ & 5.5 \\
$\mathrm{EC}(1: 2.5) \mathrm{dS} \mathrm{m}^{-1}$ & 0.09 \\
Organic carbon $(\%)$ & 1.38 \\
Calcium carbonate $(\%)$ & 1.50 \\
Available N $\left(\mathrm{kg} \mathrm{ha}^{-1}\right)$ & 486 \\
Available P $\left(\mathrm{kg} \mathrm{ha}^{-1}\right)$ & 5.20 \\
Available K $\left(\mathrm{kg} \mathrm{ha}^{-1}\right)$ & 235 \\
Fe $\left(\mathrm{mg} \mathrm{kg}^{-1}\right)$ & 8.46 \\
$\mathrm{Mn}\left(\mathrm{mg} \mathrm{kg}^{-1}\right)$ & 44.20 \\
$\mathrm{Zn}\left(\mathrm{mg} \mathrm{kg}^{-1}\right)$ & 0.40 \\
$\mathrm{Cu}\left(\mathrm{mg} \mathrm{kg}^{-1}\right)$ & 2.42 \\
\hline
\end{tabular}

Table 2. Effect of phytase and phosphatic fertilizer on availability of phosphorus

a) At 0 days of incubation

\begin{tabular}{|c|c|c|c|c|c|}
\hline \multirow{3}{*}{$\overbrace{\text { Phytase (IU) }}^{\text {Phosphorus }\left(\mathrm{kg} \mathrm{ha}^{-1}\right)}$} & \multicolumn{5}{|c|}{$P\left(k g ~ h a^{-1}\right)$} \\
\hline & \multirow{2}{*}{$\begin{array}{c}\mathbf{0} \\
4.99\end{array}$} & \multirow{2}{*}{$\begin{array}{c}\mathbf{2 5} \\
5.97 \\
\end{array}$} & \multirow{2}{*}{$\begin{array}{c}\mathbf{5 0} \\
7.56 \\
\end{array}$} & \multirow{2}{*}{\begin{tabular}{|l|}
$\mathbf{1 0 0}$ \\
8.98 \\
\end{tabular}} & \multirow{2}{*}{$\begin{array}{c}\text { Mean } \\
6.87 \\
\end{array}$} \\
\hline & & & & & \\
\hline 240 & 9.55 & 10.83 & 11.26 & 11.40 & 10.76 \\
\hline 480 & 12.25 & 12.26 & 13.11 & 14.39 & 13.00 \\
\hline 720 & 14.86 & 15.96 & 16.67 & 17.10 & 16.14 \\
\hline Mean & 10.40 & 11.25 & 12.15 & 12.98 & \\
\hline & $\mathbf{P}$ & & $\mathbf{S}$ & & \\
\hline S.E. \pm & 0.152 & & 0.152 & & \\
\hline $\mathrm{CD}$ at $\overline{5 \%}$ & 0.450 & & 0.450 & & \\
\hline
\end{tabular}

Initial soil available P: $5.20 \mathrm{~kg} \mathrm{ha}^{-1}$

b) At $15^{\text {th }}$ days of incubation

\begin{tabular}{|c|c|c|c|c|c|}
\hline \multirow{2}{*}{$\underbrace{\text { Phosphorus }\left(\mathrm{kg} \mathrm{ha}^{-1}\right)}_{\text {Phytase (IU) }}$} & \multicolumn{5}{|c|}{$\mathrm{kg} \mathrm{ha}^{-1}$} \\
\hline & $\mathbf{0}$ & 25 & 50 & 100 & Mean \\
\hline 0 & 4.96 & 12.11 & 12.53 & 16.96 & 11.64 \\
\hline 240 & 23.80 & 24.79 & 24.79 & 25.70 & 25.34 \\
\hline 480 & 27.08 & 30.07 & 34.20 & 35.34 & 34.16 \\
\hline 720 & 37.05 & 38.76 & 39.05 & 41.47 & 39.08 \\
\hline Mean & 23.97 & 27.46 & 28.58 & 30.21 & \\
\hline & \multicolumn{2}{|l|}{$\mathbf{P}$} & $\mathbf{S}$ & \multicolumn{2}{|c|}{ PxS } \\
\hline S.E. \pm & \multicolumn{2}{|l|}{0.586} & 0.586 & \multicolumn{2}{|c|}{1.446} \\
\hline $\mathrm{CD}$ at $\overline{5 \%}$ & \multicolumn{2}{|l|}{1.748} & 1.748 & \multicolumn{2}{|c|}{3.496} \\
\hline
\end{tabular}


c) At $30^{\text {th }}$ days of incubation

\begin{tabular}{|c|c|c|c|c|c|}
\hline \multirow{2}{*}{$\begin{array}{c}\text { Phosphorus }\left(\mathrm{kg} \mathrm{ha}^{-1}\right) \\
\text { Phytase (IU) }\end{array}$} & \multicolumn{5}{|c|}{$\mathrm{kg} \mathrm{ha}^{-1}$} \\
\hline & $\mathbf{0}$ & 25 & 50 & 100 & Mean \\
\hline 0 & 4.81 & 44.18 & 49.02 & 51.86 & 37.47 \\
\hline 240 & 43.70 & 51.01 & 53.15 & 47.31 & 48.79 \\
\hline 480 & 49.59 & 51.58 & 52.45 & 54.29 & 51.98 \\
\hline 720 & 48.69 & 49.59 & 51.73 & 52.87 & 50.45 \\
\hline \multirow[t]{2}{*}{ Mean } & 36.70 & 48.84 & 51.59 & 51.58 & \\
\hline & \multicolumn{2}{|l|}{$\mathbf{P}$} & \multicolumn{2}{|l|}{ S } & $\mathbf{P} \times S$ \\
\hline S.E. \pm & \multirow{2}{*}{\multicolumn{2}{|c|}{0.849}} & \multicolumn{2}{|l|}{0.849} & 1.698 \\
\hline $\mathrm{CD}$ at $\overline{5 \%}$ & & 2.777 & \multicolumn{2}{|l|}{2.777} & 5.554 \\
\hline \multicolumn{6}{|l|}{ d) At $45^{\text {th }}$ days of incubation } \\
\hline Phosphorus $\left(\mathrm{kg} \mathrm{ha}^{-1}\right)$ & \multicolumn{5}{|c|}{$\mathrm{kg} \mathrm{ha}^{-1}$} \\
\hline Phytase (IU) & $\mathbf{0}$ & 25 & 50 & 100 & Mean \\
\hline 0 & 4.81 & 44.18 & 49.02 & 51.86 & 37.47 \\
\hline 240 & 43.70 & 51.01 & 53.15 & 47.31 & 48.79 \\
\hline 480 & 49.59 & 51.58 & 52.45 & 54.29 & 51.98 \\
\hline 720 & 48.69 & 49.59 & 51.73 & 52.87 & 50.45 \\
\hline \multirow[t]{2}{*}{ Mean } & 36.70 & 48.84 & 51.59 & 51.58 & \\
\hline & \multicolumn{2}{|l|}{$\mathbf{P}$} & \multicolumn{2}{|l|}{$\mathbf{S}$} & P x S \\
\hline S.E. \pm & \multirow{2}{*}{$\begin{array}{l}0.849 \\
2.777 \\
\end{array}$} & & \multicolumn{2}{|l|}{0.849} & \multirow[t]{2}{*}{$\begin{array}{l}1.698 \\
5.554\end{array}$} \\
\hline $\mathrm{CD}$ at $\overline{5 \%}$ & & & 2.777 & & \\
\hline \multicolumn{6}{|l|}{ e) At $60^{\text {th }}$ days of incubation } \\
\hline \multirow{2}{*}{$\underbrace{\text { Phosphorus }\left(\mathrm{kg} \mathrm{ha}^{-1}\right)}_{\text {Phytase (IU) }}$} & \multicolumn{5}{|c|}{$\mathrm{kg} \mathrm{ha}^{-1}$} \\
\hline & 0 & 25 & 50 & 100 & Mean \\
\hline 0 & 4.84 & 38.05 & 41.33 & 46.03 & 32.56 \\
\hline 240 & 38.47 & 46.03 & 50.65 & 38.62 & 43.44 \\
\hline 480 & 44.60 & 49.16 & 51.30 & 52.72 & 49.44 \\
\hline 720 & 41.90 & 44.46 & 47.45 & 48.73 & 45.64 \\
\hline \multirow[t]{2}{*}{ Mean } & 32.45 & 44.42 & 47.68 & 46.52 & \\
\hline & \multicolumn{2}{|l|}{$\mathbf{P}$} & \multicolumn{2}{|l|}{$\mathbf{S}$} & $P \times S$ \\
\hline S.E. \pm & \multicolumn{2}{|l|}{0.76} & \multicolumn{2}{|l|}{0.76} & \\
\hline $\mathrm{CD}$ at $\overline{5} \%$ & 2.30 & & 2.30 & & \\
\hline
\end{tabular}

f) At $90^{\text {th }}$ days of incubation

\begin{tabular}{|c|c|c|c|c|c|}
\hline \multirow{2}{*}{$\begin{array}{l}\text { Phosphorus }\left(\mathrm{kg} \mathrm{ha}^{-1}\right) \\
\text { Phytase (IU) }\end{array}$} & \multicolumn{5}{|c|}{$\mathrm{kg} \mathrm{ha}^{-1}$} \\
\hline & 0 & 25 & 50 & 100 & Mean \\
\hline 0 & 4.90 & 39.04 & 42.04 & 45.60 & 32.89 \\
\hline 240 & 38.47 & 46.03 & 50.45 & 41.18 & 44.03 \\
\hline 480 & 46.45 & 47.31 & 48.31 & 50.16 & 48.06 \\
\hline 720 & 43.89 & 44.32 & 46.65 & 46.88 & 45.43 \\
\hline \multirow[t]{2}{*}{ Mean } & 33.43 & 44.17 & 46.86 & 45.95 & \\
\hline & $\mathbf{P}$ & & $\mathbf{S}$ & & \\
\hline S.E. \pm & 0.064 & & 0.064 & & \\
\hline $\mathrm{CD}$ at $\overline{5} \%$ & 0.192 & & 0.192 & & \\
\hline
\end{tabular}


Table 3. Effect of phytase on soil available P throughout incubation period

\begin{tabular}{|c|c|c|c|c|c|c|c|}
\hline \multirow{2}{*}{$\begin{array}{l}\text { Phytase } \\
\text { enzyme } \\
\text { (IU) }\end{array}$} & \multicolumn{6}{|c|}{$\begin{array}{l}\text { Available } \mathrm{P} \mathrm{kg} \mathrm{ha}^{-1} \\
\text { Incubation days }\end{array}$} & \multirow[t]{2}{*}{ Mean } \\
\hline & 0 & 15 & 30 & 45 & 60 & 90 & \\
\hline 0 & 6.87 & 9.19 & 11.64 & 37.47 & 32.56 & 32.89 & 21.77 \\
\hline 240 & 10.76 & 18.42 & 25.34 & 48.79 & 43.44 & 44.03 & 31.80 \\
\hline 480 & 13.00 & 25.90 & 34.16 & 51.98 & 49.44 & 48.06 & 37.09 \\
\hline 720 & 16.14 & 33.17 & 39.08 & 50.45 & 45.64 & 45.43 & 38.32 \\
\hline Mean & 11.69 & 21.67 & 27.56 & 47.17 & 42.77 & 42.60 & \\
\hline
\end{tabular}

Table 4. Effect of phosphatic fertilizer on soil available throughout incubation period

\begin{tabular}{cccccccc}
\hline $\begin{array}{c}\mathbf{P}_{\mathbf{2}} \mathbf{O}_{\mathbf{5}} \text { levels } \\
\left(\mathbf{k g ~ h a}^{-\mathbf{1}} \mathbf{)}\right.\end{array}$ & \multicolumn{9}{c}{$\begin{array}{c}\text { Available P } \mathbf{~ k g ~ h a} \\
\text { Incubation days }\end{array}$} & \multirow{2}{*}{ Mean } \\
\cline { 2 - 6 } & $\mathbf{0}$ & $\mathbf{1 5}$ & $\mathbf{3 0}$ & $\mathbf{4 5}$ & $\mathbf{6 0}$ & $\mathbf{9 0}$ & \\
\hline 0 & 10.40 & 18.10 & 23.97 & 36.70 & 32.45 & 33.43 & 25.84 \\
25 & 11.25 & 20.84 & 27.46 & 48.84 & 44.42 & 44.17 & 32.83 \\
50 & 12.15 & 22.83 & 28.58 & 51.59 & 47.68 & 46.86 & 34.95 \\
100 & 12.98 & 24.90 & 30.21 & 51.58 & 46.52 & 45.95 & 35.36 \\
Mean & 11.70 & 21.67 & 27.56 & 47.18 & 42.77 & 42.60 & \\
\hline
\end{tabular}

Table 5. Effect of phytase and phosphatic fertilizer levels on cumulative available phosphorus

\begin{tabular}{|c|c|c|c|c|c|c|c|}
\hline \multirow{2}{*}{ Treatments } & \multicolumn{6}{|c|}{ Incubation days } & \multirow{2}{*}{$\begin{array}{l}\text { Cumulative soil } \\
\text { available } \mathbf{P} \\
\left(\mathrm{kg} \mathrm{ha}^{-1}\right)\end{array}$} \\
\hline & $\mathbf{0}$ & 15 & 30 & 45 & 60 & 90 & \\
\hline $\mathbf{P}_{0} \mathbf{S}_{0}$ & 4.99 & 4.84 & 4.96 & 4.81 & 4.84 & 4.9 & 29.34 \\
\hline $\mathbf{P}_{0} \mathbf{S}_{1}$ & 5.97 & 8.69 & 12.11 & 44.18 & 38.05 & 39.04 & 148.04 \\
\hline $\mathbf{P}_{0} \mathbf{S}_{2}$ & 7.56 & 10.12 & 12.53 & 49.02 & 41.33 & 42.04 & 162.60 \\
\hline $\mathbf{P}_{0} \mathbf{S}_{3}$ & 8.98 & 13.11 & 16.96 & 51.86 & 46.03 & 45.60 & 182.54 \\
\hline $\mathbf{P}_{1} \mathbf{S}_{0}$ & 9.55 & 15.25 & 23.80 & 43.70 & 38.47 & 38.47 & 169.24 \\
\hline $\mathbf{P}_{1} \mathbf{S}_{1}$ & 10.83 & 17.24 & 24.79 & 51.01 & 46.03 & 46.03 & 195.93 \\
\hline $\mathbf{P}_{1} \mathbf{S}_{2}$ & 11.26 & 19.09 & 24.79 & 53.15 & 50.65 & 50.45 & 209.39 \\
\hline $\mathbf{P}_{1} \mathbf{S}_{3}$ & 11.40 & 22.09 & 25.70 & 47.31 & 38.62 & 41.18 & 186.30 \\
\hline $\mathbf{P}_{2} \mathbf{S}_{0}$ & 12.25 & 23.94 & 27.08 & 49.59 & 44.60 & 46.45 & 203.91 \\
\hline $\mathbf{P}_{2} \mathbf{S}_{1}$ & 12.26 & 26.36 & 30.07 & 51.58 & 49.16 & 47.31 & 216.74 \\
\hline $\mathbf{P}_{2} \mathbf{S}_{2}$ & 13.11 & 27.21 & 34.20 & 52.45 & 51.30 & 48.31 & 226.58 \\
\hline $\mathbf{P}_{2} \mathbf{S}_{3}$ & 14.39 & 26.08 & 35.34 & 54.29 & 52.72 & 50.16 & 232.98 \\
\hline $\mathbf{P}_{3} \mathbf{S}_{\mathbf{0}}$ & 14.86 & 28.35 & 37.07 & 48.69 & 41.90 & 43.89 & 214.76 \\
\hline $\mathbf{P}_{3} \mathbf{S}_{1}$ & 15.96 & 31.07 & 38.76 & 49.59 & 44.46 & 44.32 & 224.16 \\
\hline $\mathbf{P}_{3} \mathbf{S}_{2}$ & 16.67 & 34.91 & 39.05 & 51.73 & 47.45 & 46.65 & 236.46 \\
\hline $\mathbf{P}_{3} \mathbf{S}_{3}$ & 17.1 & 38.33 & 41.47 & 52.87 & 48.73 & 48.73 & 247.23 \\
\hline S.E \pm & 0.304 & 1.377 & 1.446 & 1.698 & 1.530 & 0.128 & \\
\hline C.D at $\overline{5} \%$ & 0.900 & 4.197 & 3.496 & 5.554 & 4.590 & 0.385 & \\
\hline
\end{tabular}


Table 6. Effect of phytase and phosphatic fertilizer levels on phosphorus release potential

\begin{tabular}{|c|c|c|c|c|c|c|}
\hline \multirow{3}{*}{$\begin{array}{c}\text { Treatment } \\
\text { combinations }\end{array}$} & \multicolumn{5}{|c|}{ Per day potential $\left(\mathrm{kg} \mathrm{ha}^{-1}\right)$} & \multirow{3}{*}{ Mean } \\
\hline & \multicolumn{5}{|c|}{ Incubation days } & \\
\hline & 15 & 30 & 45 & 60 & 90 & \\
\hline $\mathbf{P}_{0} \mathbf{S}_{0}$ & -0.01 & 0.00 & 0.00 & 0.00 & 0.00 & 0.00 \\
\hline $\mathbf{P}_{0} \mathbf{S}_{1}$ & 0.18 & 0.20 & 0.85 & 0.53 & 0.37 & 0.36 \\
\hline $\mathbf{P}_{0} \mathbf{S}_{2}$ & 0.17 & 0.17 & 0.92 & 0.56 & 0.38 & 0.37 \\
\hline $\mathrm{P}_{0} \mathrm{~S}_{3}$ & 0.28 & 0.27 & 0.95 & 0.62 & 0.41 & 0.42 \\
\hline $\mathbf{P}_{1} \mathbf{S}_{0}$ & 0.38 & 0.48 & 0.76 & 0.48 & 0.32 & 0.40 \\
\hline $\mathbf{P}_{1} \mathbf{S}_{1}$ & 0.43 & 0.47 & 0.89 & 0.59 & 0.39 & 0.46 \\
\hline $\mathbf{P}_{1} \mathbf{S}_{2}$ & 0.52 & 0.45 & 0.93 & 0.66 & 0.44 & 0.50 \\
\hline $\mathbf{P}_{1} \mathbf{S}_{3}$ & 0.71 & 0.48 & 0.80 & 0.45 & 0.33 & 0.46 \\
\hline $\mathbf{P}_{2} \mathbf{S}_{0}$ & 0.78 & 0.49 & 0.83 & 0.54 & 0.38 & 0.50 \\
\hline $\mathbf{P}_{2} \mathbf{S}_{1}$ & 0.94 & 0.59 & 0.87 & 0.62 & 0.39 & 0.57 \\
\hline $\mathbf{P}_{2} \mathbf{S}_{2}$ & 0.94 & 0.70 & 0.87 & 0.64 & 0.39 & 0.59 \\
\hline $\mathbf{P}_{2} \mathbf{S}_{3}$ & 0.78 & 0.70 & 0.89 & 0.64 & 0.40 & 0.57 \\
\hline $\mathbf{P}_{3} \mathbf{S}_{0}$ & 0.90 & 0.74 & 0.75 & 0.45 & 0.32 & 0.53 \\
\hline $\mathbf{P}_{3} \mathbf{S}_{1}$ & 1.01 & 0.76 & 0.75 & 0.48 & 0.32 & 0.55 \\
\hline $\mathbf{P}_{3} \mathbf{S}_{2}$ & 1.22 & 0.75 & 0.78 & 0.00 & 0.00 & 0.46 \\
\hline $\mathbf{P}_{3} \mathbf{S}_{3}$ & 1.42 & 0.81 & 0.79 & 0.53 & 0.37 & 0.65 \\
\hline Mean & 0.67 & 0.50 & 0.79 & 0.49 & 0.33 & \\
\hline
\end{tabular}

\section{References:}

Aseri, G. K., Jain, N. and Tarafdar, J. C. (2009). Hydrolysis of organic phosphate forms by phosphatases and phytate producing fungi of arid and semi arid soils of India. AmericanEurasian Journal of Agriculture and Environment Science 5, 564-570.

Bray, R. H. and Kurtz, L. T. (1945). Determination of total, organic, and available forms of Phosphorus in soils. Soil Science 59, 39-45.

Coutinho, F. P., Felix, W.P. and Yano-Melo, A. M. (2012). Solubilization of phosphates in vitro by Aspergillus spp. and Penicillium spp. Ecological Engineering 42, 85-89.

Gujar, P. D., Bhavsar, K. P. and Khire, J. M. (2013). Effect of phytase from Aspergillus niger on plant growth and mineral assimilation in wheat (Triticum aestivum Linn.) and its potential for use as a soil amendment. Journal of Science Food Agriculture 93, 2242-2247. 1
Jagdish Prasad and Ram, H. (1986). Effect of zinc and copper and Rhizobium inoculation on phosphorus availability and uptake in mungbean. Journal of the Indian Society of Soil Science 34,762-766.

Subba Rao, N. S. (1995). Soil Microorganisms and Plant Growth. (Third Edition). Science Publishers, Inc.

Whitelaw, M. A. (1999). Growth promotion of plants inoculated with phosphate solubilizing fungi. Advances in Agronomy 69, 99-151.

Yadav, R. S. and Tarafdar, J. C., (2003). Phytase and phosphatases producing fungi in arid and semi arid soils and their efficiency in hydroloyzing different organic P compounds. Soil Biology and Biochemistry 35, 745-751.

Yadav, B. K. and Verma, A. (2012). Phosphate solubilisation and mobilization in soil through microorganisms under arid ecosystem. The Functioning of Ecosystems. (Ed. M. Ali) pp. 93108. 